\title{
An optical time-delay for the lensed BAL quasar HE 2149-2745^
}

\author{
I. Burud ${ }^{1,2}$, F. Courbin ${ }^{1,3,4}$, P. Magain ${ }^{1}$, C. $\operatorname{Lidman}^{5}$, D. Hutsemékers ${ }^{5, \star \star}$, J.-P. Kneib ${ }^{6}$, J. Hjorth ${ }^{7}$, \\ J. Brewer ${ }^{5}$, E. Pompei ${ }^{5}$, L. Germany ${ }^{5}$, J. Pritchard ${ }^{5}$, A. O. Jaunsen ${ }^{5}$, G. Letawe ${ }^{1}$, and G. Meylan ${ }^{2}$ \\ 1 Institut d'Astrophysique et de Géophysique, Université de Liège, Avenue de Cointe 5, 4000 Liège, Belgium \\ 2 Space Telescope Science Institute, 3700 San Martin Drive, Baltimore, MD 21218, USA \\ 3 Universidad Católica de Chile, Departamento de Astronomia y Astrofisica, Casilla 306, Santiago 22, Chile \\ 4 GEPI, Observatoire de Paris-Meudon, Place Jules Janssen, 92195 Meudon, France \\ 5 European Southern Observatory, Casilla 19, Santiago, Chile \\ 6 Observatoire Midi-Pyrénées (LAS), UMR 5572, 14 avenue E. Belin, 31400 Toulouse, France \\ 7 Astronomical Observatory, University of Copenhagen, Juliane Maries Vej 30, 2100 Copenhagen $\varnothing$, Denmark
}

Received 1 June 2001 / Accepted 26 November 2001

\begin{abstract}
We present optical $V$ and $i$-band light curves of the gravitationally lensed BAL quasar HE 2149-2745. The data, obtained with the $1.5 \mathrm{~m}$ Danish Telescope (ESO-La Silla) between October 1998 and December 2000, are the first from a long-term project aimed at monitoring selected lensed quasars in the Southern Hemisphere. A time delay of $103 \pm 12$ days is determined from the light curves. In addition, VLT/FORS1 spectra of HE 2149-2745 are deconvolved in order to obtain the spectrum of the faint lensing galaxy, free of any contamination by the bright nearby two quasar images. By cross-correlating the spectrum with galaxy-templates we obtain a tentative redshift estimate of $z=0.495 \pm 0.01$. Adopting this redshift, a $\Omega=0.3, \Lambda=0.7$ cosmology, and a chosen analytical lens model, our time-delay measurement yields a Hubble constant of $H_{0}=66 \pm 8 \mathrm{~km} \mathrm{~s}^{-1} \mathrm{Mpc}^{-1}$ ( $1 \sigma$ error) with an estimated systematic error of $\pm 3 \mathrm{~km} \mathrm{~s}^{-1} \mathrm{Mpc}^{-1}$. Using non-parametric models yields $H_{0}=65 \pm 8 \mathrm{~km} \mathrm{~s}^{-1} \mathrm{Mpc}^{-1}$ ( $1 \sigma$ error) and confirms that the lens exhibits a very dense/concentrated mass profile. Finally, we note, as in other cases, that the flux ratio between the two quasar components is wavelength dependent. While the flux ratio in the broad emission lines - equal to 3.7 - remains constant with wavelength, the continuum of the brighter component is bluer. Although the data do not rule out extinction of one quasar image relative to the other as a possible explanation, the effect could also be produced by differential microlensing by stars in the lensing galaxy.
\end{abstract}

Key words. gravitational lensing - quasars: individual: HE 2149-2745 - cosmological parameters

\section{Introduction}

The time-delay between the gravitationally lensed images of a distant source is a measurable parameter. Observed as the time difference between the arrival dates of a single (lensed) wavefront emitted by a distant source, it is directly related to the Hubble constant $H_{0}$ (Refsdal 1964). Obtaining accurate time-delay measurements in multiply lensed quasars can therefore yield (i) a determination of $H_{0}$ provided the mass distribution in the lens is known, or (ii) constraints on the mass distribution in

Send offprint requests to: I. Burud, e-mail: burud@stsci.edu

* Based on observations made with the Danish 1.5-m telescope (ESO, La Silla, Chile) and at VLT UT1 Antu (ESOParanal, Chile) (Proposals: 64.O-0205(B), 65.O-0214(B), 66.A0203(B), 67.A-0115(B), 66.B-0139(A) and HST archives ID $8175)$.

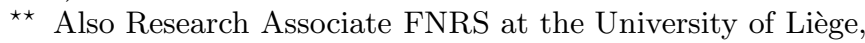
Belgium. a given lens, using $H_{0}$ as inferred from other methods. During the last 20 years much effort has been devoted to the observations of lensed quasars, and in particular to long-term monitoring of selected systems. Some of these are Q0957+561 (Schild 1990; Vanderriest et al. 1989), PG 1115+080 (Schechter et al. 1997), B1608+656 (Fassnacht et al. 1999) and B1600+434 (Burud et al. 2000; Koopmans et al. 2000). In this context, we have been conducting a photometric monitoring program at the Danish 1.5-m telescope at La Silla observatory (ESO, Chile) since October 1998, with the goal of measuring the time-delays in several well studied lensed quasars. We present here the first result from this program: the time-delay measurement in the two-image quasar HE 2149-2745.

The lensed nature of the BAL quasar HE 2149-2745 at $z=2.03$ was established by Wisotzki et al. (1996). This system proves to be an easy target for monitoring at a site with reasonable seeing conditions (up to $2^{\prime \prime}$ ): it is bright $(B=17.3)$ and the two quasar images have an 


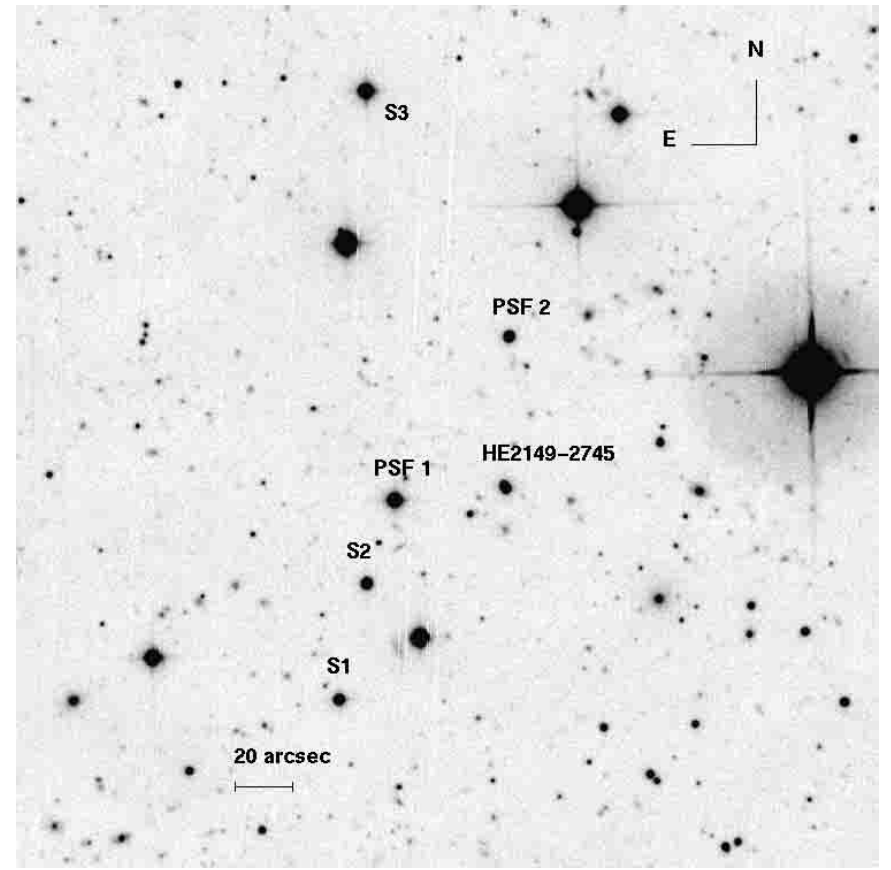

Fig. 1. Field of view $5^{\prime} \times 5^{\prime}$ in size around HE 2149-2745. The three reference stars (labelled S1, S2, S3) used for the photometry and the two PSF-stars (PSF1, PSF2) used in the spectral deconvolution are indicated. North is up and East to the left.

angular separation of $1.7^{\prime \prime}$. The monitoring program, the light curves and the time delay are discussed in Sects. 2-4 below.

The lensing galaxy has been detected in HST NICMOS and WFPC2 images but its redshift remains unknown. With the aim of measuring this redshift, we have obtained a spectrum of HE 2149-2745 with FORS1 at UT1 (ESOParanal, Chile). The analysis of the spectroscopic data is described in Sect. 5. This section also includes a discussion on the spectral differences between the two quasar components. Mass models and estimates of the Hubble constant are presented in Sect. 6. Finally, Sect. 7 summarises the main results.

\section{Photometric monitoring at the $\mathbf{1 . 5} \mathbf{m}$ Danish telescope}

\subsection{Observations and data reduction}

Weekly observations of HE 2149-2745 were carried out at the Danish 1.5-m telescope at ESO-La Silla from October 1998 to December 2000. The target is visible from the beginning of June to the end of December, which results in gaps of about $\sim 5$ months in the light curves. Apart from these gaps, very few points were missed because of poor weather or technical problems. Observations were obtained in the $V$ and Gunn $i$ bands with DFOSC (Danish Faint Object Spectrograph Camera) which has a pixel size of 0.395 . Figure 1 shows the central region of the field of view of DFOSC, i.e., $5 \mathrm{arcmin}^{2}$ of the $13 \mathrm{arcmin}^{2}$ available. The $V$-band was given priority because of the

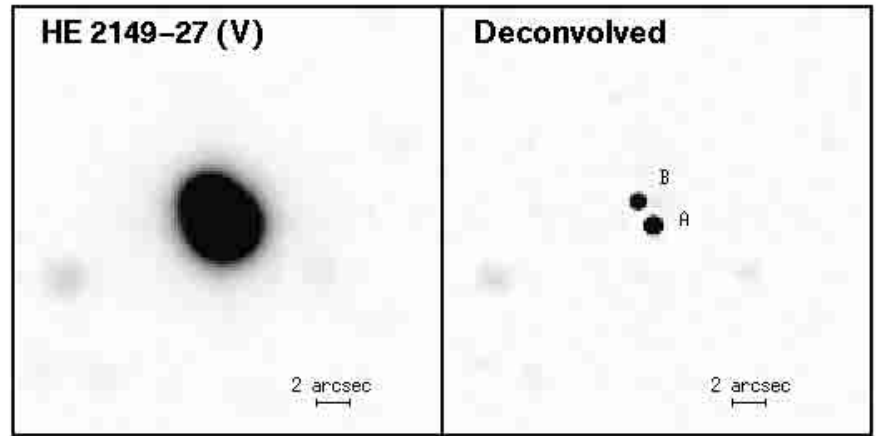

Fig. 2. Left: stacked $V$-band image of a $9^{\prime \prime} \times 9^{\prime \prime}$ region centered on HE $2149-2745$. The seeing is $\sim 2^{\prime \prime}$ and the total exposure time is $\sim 17$ hours. Right: deconvolved image ( $\left.F W H M=00^{\prime \prime} 4\right)$ obtained from the simultaneous deconvolution of 57 frames. North is up and East is to the left.

Table 1. Positions of three reference stars relative to the position of the A component in HE 2149-2745.

\begin{tabular}{ccc}
\hline Star & RA $(\operatorname{arcsec})$ & DEC $(\operatorname{arcsec})$ \\
\hline S1 & -48.126 & 136.268 \\
S2 & -57.126 & -72.647 \\
S3 & -38.160 & -4.145 \\
\hline
\end{tabular}

detector's better sensitivity at these wavelengths, but observations were also carried out in the $i$-band in order to monitor possible colour changes. The exposure time was set to $900 \mathrm{~s}$ during the first 6 months of observations. It was increased to $1800 \mathrm{~s}$ for the rest of the observations in order to improve the photometric quality for the faint B quasar image. For each observed data point the total exposure time was divided into three dithered exposures in order to remove cosmic rays and bad pixels. The seeing varied from 0 .' 7 to 2 ". 9 , with a median of $1^{\prime \prime} 6$. All the imaging data were pre-processed (bias-subtracted and flat-field corrected) using standard IRAF routines.

\section{Photometry}

The photometry of the blended quasar images was performed by applying the MCS deconvolution algorithm (Magain et al. 1998). This algorithm has already been used to analyze the data of several lensed quasars (e.g., Burud et al. 2000; Hjorth et al. 2001). Its main advantage is its ability to use all the data, even rather poor, irrespective of image quality and lunar phase. The final deconvolved image is produced by simultaneously deconvolving all the individual frames of the same object from all epochs. The positions of the quasar images (two in the present case) and the shape of the lensing galaxy are the same for all the images and are therefore constrained using the total $S / N$ of the whole data-set. The intensity of the point sources are allowed to vary from image to image, hence producing the light curves.

The two quasar components are well separated in our deconvolved image of HE 2149-2745 (see Fig. 2), but the lensing galaxy remains too faint to be detected. This is 

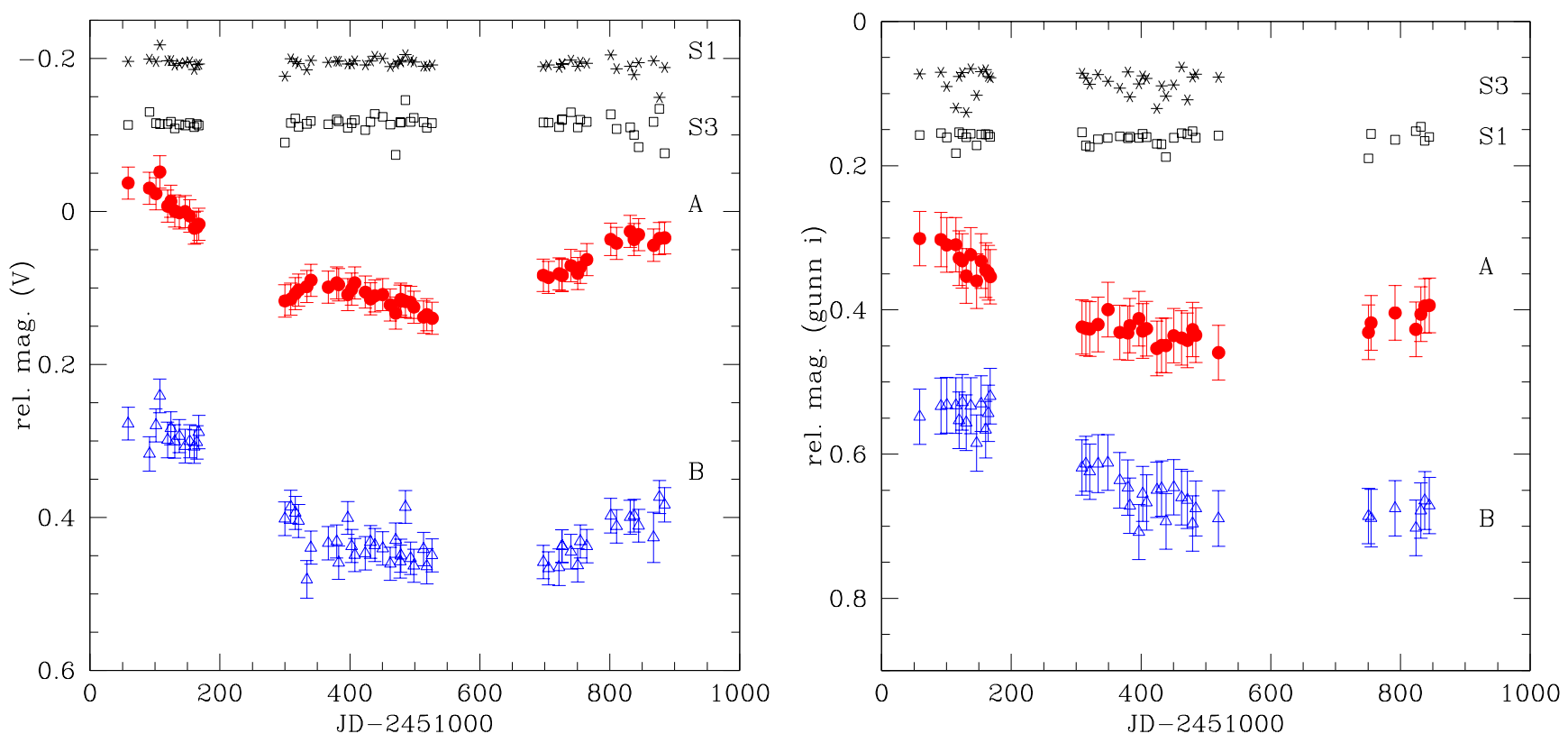

Fig. 3. Light curves of the two images A and B of HE 2149-2745 and the comparison stars S1 and S3 (Table 1). The V-band light curves are displayed on the left while the Gunn $i$-band light curves are shown on the right. The magnitudes of the quasar and stars are calculated relative to the reference star S2. The error bars include photon noise and PSF errors estimated from the deconvolution of a reference star. For display purposes, the B component is shifted by -1.3 mag and -1.2 mag in the $V$ and Gunn $i$ band, respectively, with respect to their original values. Likewise, S1 is shifted by 1.1 and 1.35 , and S3 is shifted by +1.15 and +1.35 mag in the $V$ and Gunn $i$ filters, respectively. S3 was saturated in the last Gunn $i$ images and is therefore not shown on the plot for these frames.

fortuitous since contamination by an extended object would complicate the analysis.

The light curves of HE $2149-2745$ consist of 57 data points in the $V$-band and 41 points in the Gunn $i$ band, as presented in Fig. 3. The points between JD 2451800 and JD 2451900 were obtained just after the installation of the new chip on DFOSC. The new chip does not have problems of charge diffusion, as the old chip had, and consequently, the effective seeing is now considerably improved. Our original PSF star, for which the ADU counts were always in the linear regime on the old CCD, became saturated on the new chip. Another PSF star further away from the target had to be used on a few frames before the exposure time was adjusted to the new chip. These points are therefore more noisy than the previous points.

The data are plotted relative to a reference star common to all images, star S2 in Table 1. Two other stars were deconvolved as well, in order to check the relative photometry and to check for systematic errors (see Table 1 for the positions of the stars). The error-bars include both photon noise and additional systematic errors, e.g., PSF errors. The latter is estimated by using one of the reference stars, as explained in Burud et al. (2000).

\section{Time-delay measurement}

By sliding the light curves across one another, one can make a rough "by eye" estimate of the time-delay of $\sim 100$ days, with $\mathrm{A}$ leading $\mathrm{B}$. Using the $\chi^{2}$ minimization method described in Burud et al. (2000), a more objective value of $\Delta t=103 \pm 12$ days is found from the $V$-band light curves. The $i$-band data are noisier and have fewer data points but the measured time-delay, $\Delta t=104 \pm$ 31 days, agrees well with the delay derived from the $V$ band. A simultaneous minimization of the $V$ and $i$ band curves gave a $\Delta t=109 \pm 22$ days. The errors quoted here are obtained from Monte Carlo simulations of 1000 sets of light curves, assuming that the photometric errors are uncorrelated and follow a Gaussian distribution. The $\chi^{2}$ minimization method was also performed on 1000 sets of curves where 5 randomly chosen data points were removed from each set. The results from these simulations yielded a time-delay of $\Delta t=101 \pm 30$ days and $\Delta t=107 \pm 30$ days in $V$ and $i$ respectively, confirming that the time-delay measurement is robust. The magnitude differences between the $\mathrm{A}$ and the $\mathrm{B}$ components are found to be $1.635 \pm 0.001$ mag in $V$ and $1.505 \pm 0.003 \mathrm{mag}$ in the $i$-band. This corresponds to flux ratios of 4.51 and 4.00 in $V$ and $i$ bands respectively Although no erratic changes are detected in the light curves within the measurement errors, microlensing on long time scales may still be present as suggested by the VLT spectra presented in Sect. 5 . Given that the $V$-band data contain more points and are less noisy than the $i$-band data, we adopt the $V$-band estimate of the time-delay as the best one: $\Delta t=103 \pm 12$ days ( $1 \sigma$ error). Figure 3 summarizes the photometric data and Fig. 4 displays the $V$ and $i$-band light curves with component B shifted by 103 days. 

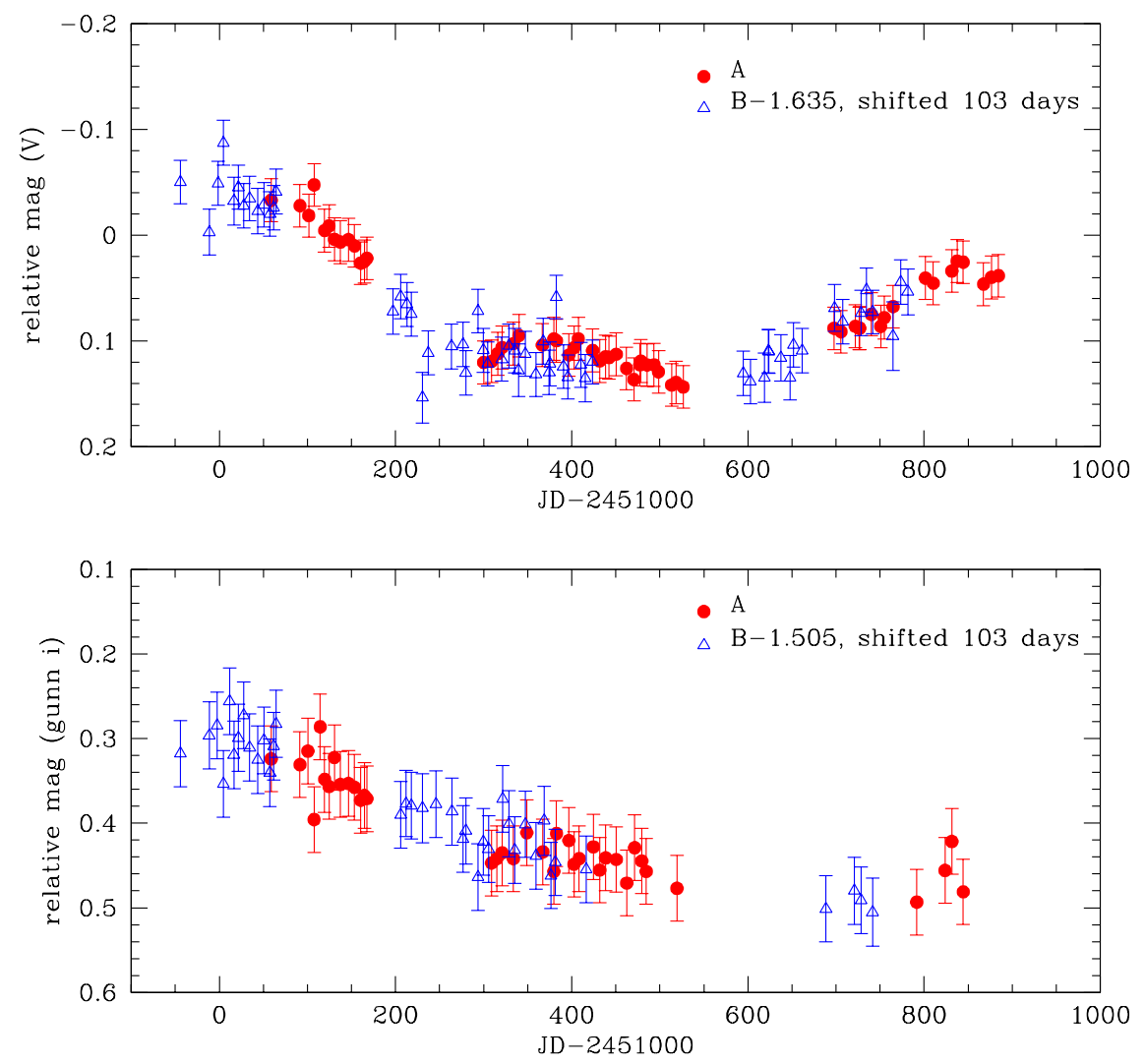

Fig. 4. The $V$ (top) and $i$-band (bottom) light curves where the B curve is shifted by 103 days. The light curve of component B is shifted by 1.635 and 1.505 magnitudes in the $V$ and $i$-band, respectively, with respect to their original values. The curves for the A component are as in Fig. 3.

\section{Spectroscopy of HE $2149-2745$}

\subsection{VLT spectroscopy}

To convert the time-delay into an estimate of the Hubble constant, one must know the geometry of the lensing system and this includes the redshift of the lensing galaxy. For this purpose, we took an optical spectrum of HE 2149-2745 with the ESO VLT/UT1 using the Multi-Object-Spectroscopy (MOS) capability of FORS1. The observations were obtained on November 19, 2000 under fairly good seeing conditions $\left(0.8^{\prime \prime}\right)$. The $1^{\prime \prime}$ slitlets of FORS1 were aligned to obtain simultaneously the spectrum of HE 2149-2745 and of 2 PSF stars (indicated in Fig. 1) about as bright as the two quasar images. The high resolution collimator was used, resulting in a pixel size of $0.1^{\prime \prime}$, in combination with the G300V grism and GG435 order sorting filter. Three exposures, each of $1000 \mathrm{~s}$, were taken so that cosmic rays could be removed.

\subsection{Redshift of the lensing galaxy}

Using the spatial information in the spectra of the two PSF stars, the spectrum was spatially deconvolved with the spectral version of the MCS deconvolution method (Courbin et al. 2000a). The deconvolution process decomposes the data into the individual spectra of the two quasar images and the faint lensing galaxy (see Figs. 5 and 7 ), in a way similar to the one described in Lidman et al. (2000) for HE 1104-1805.

The lens spectrum was cross-correlated with a template spectrum of an elliptical galaxy (Kinney et al. 1996). Due to the low $S / N$ the calculated correlations are low, nevertheless, there is a significant "bump" in the correlation function covering the redshift range $0.49 \leq z \leq 0.60$ as shown in Fig. 6. The spectrum of the lens and the template are shown in Fig. 5, where the template has been shifted vertically for clarity. The calcium $\mathrm{H}$ and $\mathrm{K}$ lines, the $G$-band and the $\mathrm{Mg}$ triplet are labeled. A measure of the reliability of the redshift estimate is given by the $r$-statistic of Tonry \& Davis (1979), which is the signalto-noise ratio of the main peak in the cross correlation. We find that $r=1.9$ meaning that the signal-to-noise of the correlation peak is poor. A value above 3 is considered secure (Kurtz \& Mink 1998).

As can be seen on the HST image (cf. Fig. 14) the lensing galaxy lies very close to the B image. We therefore looked for evidence of absorption in the spectrum of the background quasar. In order to remove the quasar spectral features, the B spectrum was divided by that of the A component (Fig. 8). Two, possible Ca H \& K candidates were found, one at $z=0.489$ and another at $z=0.504$. An integrated $\mathrm{A}+\mathrm{B}$ spectrum at similar resolution but covering shorter wavelengths has been published by Wisotzki et al. (1996). We looked for possible MgII absorption in 


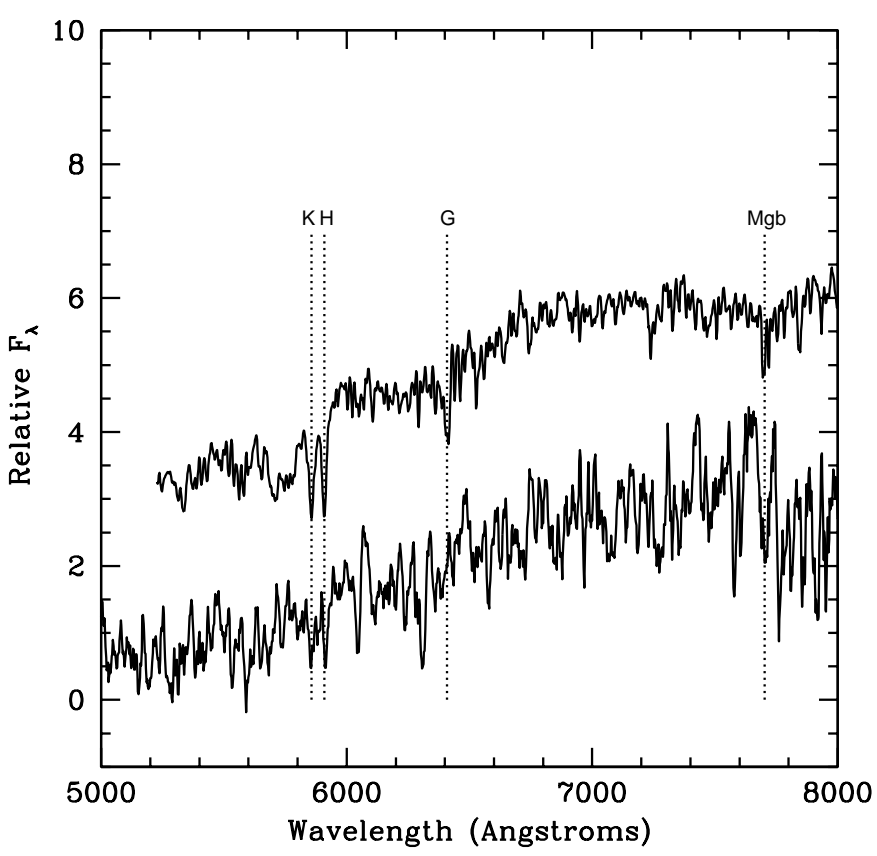

Fig. 5. Extracted spectrum of the lensing galaxy (bottom) and the template spectrum from Kinney et al. (1996) (top) used in the cross correlation. The main spectral features, calcium $\mathrm{H}$ and $\mathrm{K}$ lines, the $G$-band and the $\mathrm{Mg}$ triplet, are indicated by dashed vertical lines. The lens redshift is tentatively $z=0.489$.

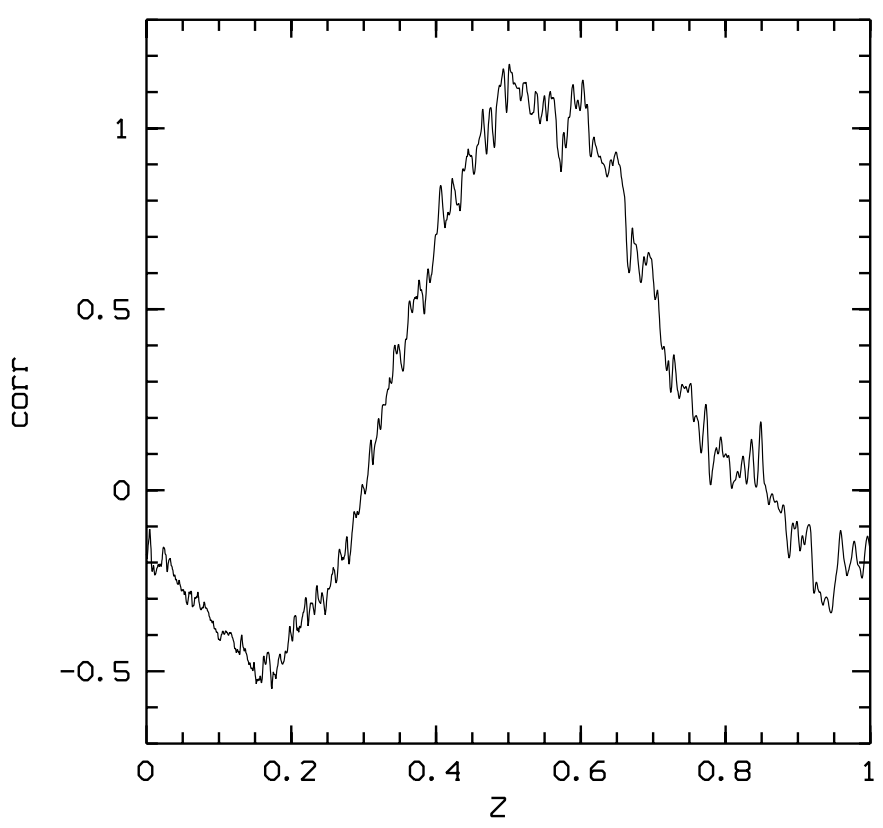

Fig. 6. Cross-correlation function of the galaxy spectrum with the galaxy template from Kinney et al. (1996). The centre of the correlation peak is at $z=0.535$.

the quasar spectrum at the putative redshift of the lens. Unfortunately, the $z=0.489$ line would fall in a strong absorption line of the quasar, while a candidate at $z=0.504$ is found in the wing of a quasar emission. The signal-tonoise ratio of the lens galaxy and the $\mathrm{Ca} \mathrm{H}$ and $\mathrm{K}$ absorption features in the $\mathrm{B}$ component are very low and we can not rule out any of the two estimates. We therefore take $z=0.495 \pm 0.01$ to be the most likely redshift for the lensing galaxy. This is within the estimated redshift range

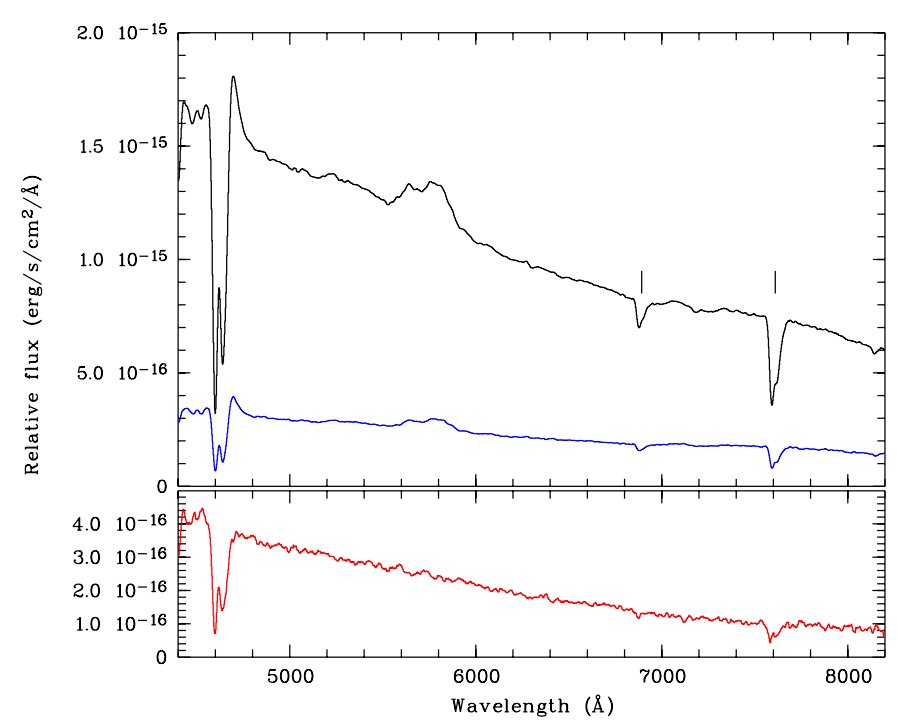

Fig. 7. Top: spectra of the A and B components. Bottom: the spectrum showing $A-3.7 \times B$. The scaling factor is chosen to best cancel the emission lines. Telluric absorption lines are indicated.

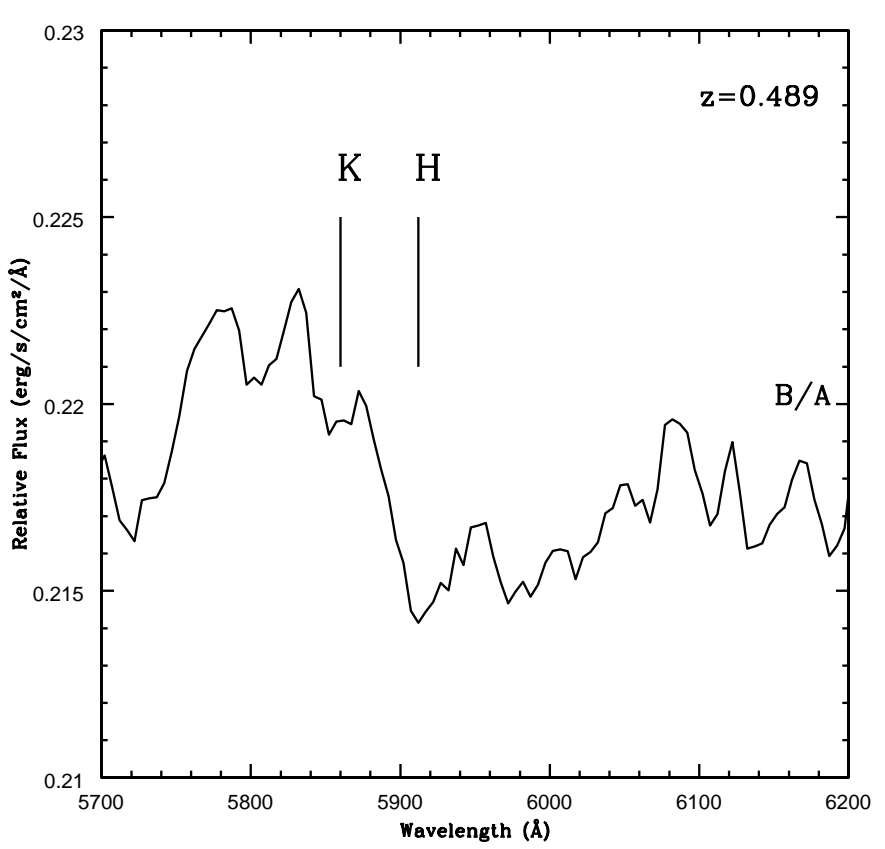

Fig. 8. Spectrum of the B component divided by that of the A component of HE 2149-2745. The plot shows a zoom on the region corresponding to possible Calcium $\mathrm{H}$ and $\mathrm{K}$ absorption due to the lens at $z=0.489$. The bump between 5750 and $5900 \AA$ is the residual CIII]+AlIII emission from the quasar.

$0.37 \leq z \leq 0.50$ which is based on the position of the lens in the fundamental plane (Kochanek et al. 2000).

\subsection{Spectroscopy of the quasar images: Colour differences, extinction or microlensing?}

The quasar spectra show that the continuum of component $\mathrm{A}$ is bluer than the continuum of component $\mathrm{B}$ (Fig. 7). This confirms the difference in flux ratio that was found from the light curves in the $V$ and Gunn 


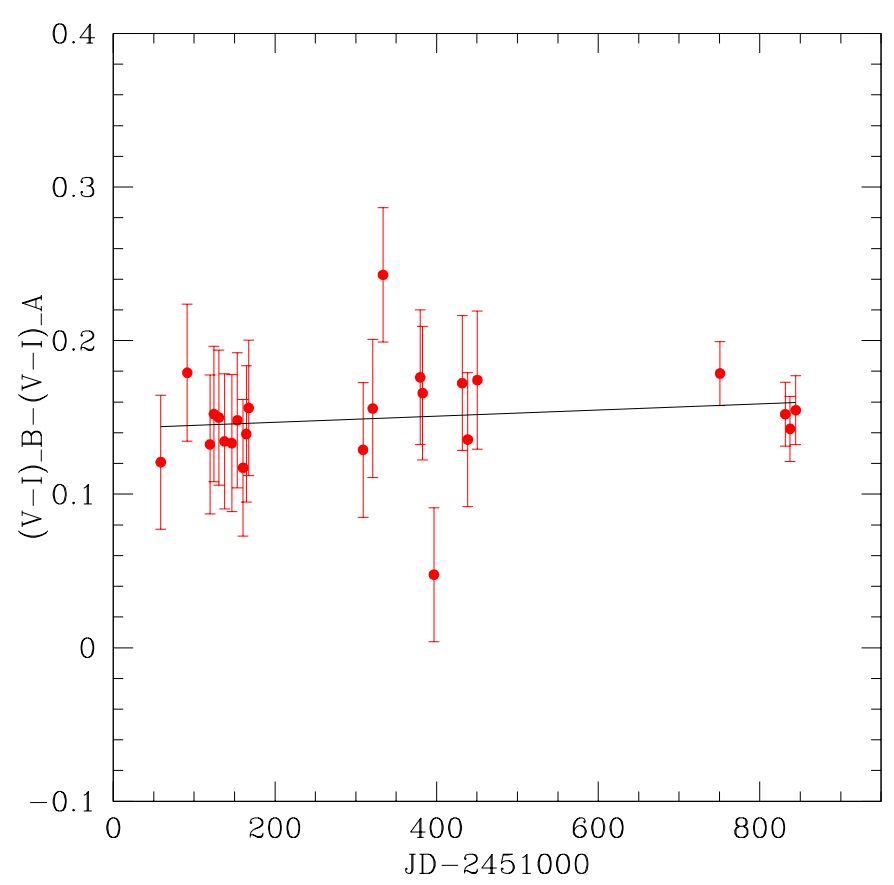

Fig. 9. Colour excess of the $\mathrm{B}$ component relative to the $\mathrm{A}$ component, $(V-I)_{\mathrm{B}}-(V-I)_{\mathrm{A}}$ as a function of time. No significant variations can be detected over a period of 900 days. The solid line shows the best fit line through the points.

$i$-bands (Sect. 4). We note three points of interest about these spectral differences:

1. As shown in Fig. 9 the colour difference between the quasar images does not vary during the period of our observations ( $\sim 3$ years);

2. The emission line flux ratios at the date of the observation are not wavelength dependent: all emission lines cancel in the difference spectrum of Fig. 7. This difference spectrum has been produced by multiplying the spectrum of the $\mathrm{B}$ component by a constant factor of 3.7 and by subtracting it from the spectrum of the A component. Figure 10 gives a different representation of the same effect, where the spectra of the two quasar images are normalized to the same continuum. The equivalent widths of all emission lines (including the FeII pseudo-continuum) are smaller in A;

3. The CIV broad absorption line behaves as the continuum: its equivalent width is unchanged in the $\mathrm{A}$ and B spectra (Fig. 10).

The spectral differences cited above have essentially two possible explanations: $(i)$ differential magnification due to microlensing, or ( $i i)$ differential reddening of the B component by the lensing galaxy.

\subsubsection{Microlensing}

If microlensing is present in HE 2149-2745, one expects the continuum region and the much more extended

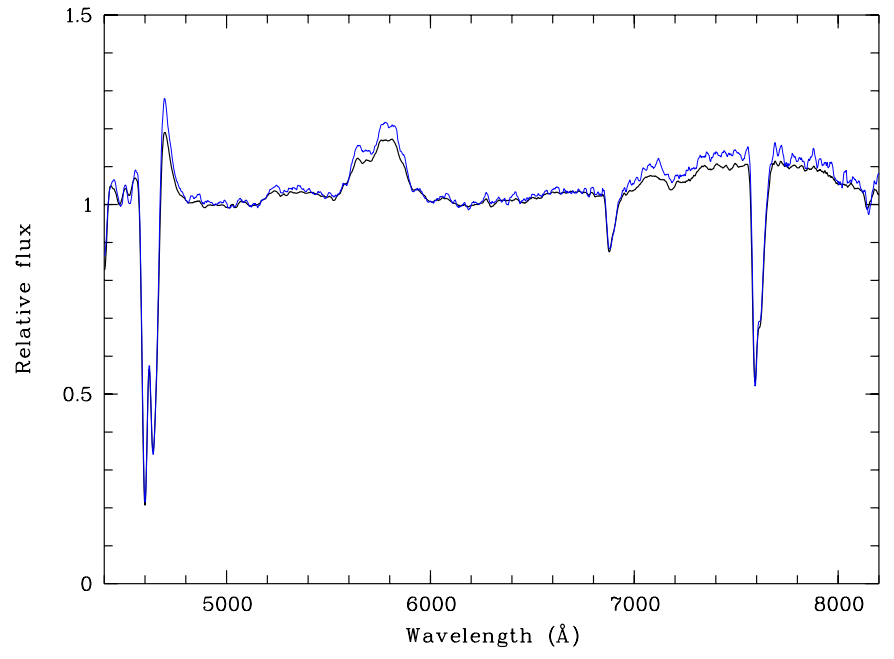

Fig. 10. The A (thick) and B (thin) spectra of HE 2149-2745 normalized to a common continuum. The equivalent widths of the emission lines, including the FeII pseudo-continuum, are smaller in A. The equivalent width of the CIV broad absorption line $(\sim 4650 \AA)$ is the same in both the $\mathrm{A}$ and $\mathrm{B}$ components.

emission lines region to be affected differently (Wambsganss \& Paczyǹski 1991). In most AGN models, the size of the continuum region depends on wavelength, and the Broad Emission Line Region (BELR) is usually believed to be larger than the continuum region by more than one order of magnitude. Since the B component is closest to the lensing galaxy we would expect that microlensing effects occur most often in the B-component. Microlensing of the A component however is also possible.

Assuming the lensed quasar in HE 2149-2745 follows the "standard" AGN model and that we are observing component A through a network of caustics produced by stars in the main lensing galaxy, one can imagine a scenario where the inner - and bluer - continuum region of the component $\mathrm{A}$ is being enhanced by a larger amount than the outer redder parts. As the BELR is much larger than the central AGN (even the redder parts), it remains unaffected by microlensing. This interpretation has already been proposed to explain similar spectral differences observed in the double HE 1104-1805 (Wisotzki et al. 1993, 1995; Courbin et al. 2000b).

In the case of HE 2149-2745 the Einstein radius of a typical deflector in the lens plane projected onto the source plane is $(\Omega=0.3, \Lambda=0)$ :

$R_{\mathrm{E}}=\sqrt{\frac{4 G M}{c^{2}} \frac{D_{\mathrm{ds}} D_{\mathrm{s}}}{D_{\mathrm{d}}}}=1.3 \times 10^{-3}\left(M / 0.1 M_{\odot}\right)^{1 / 2} \mathrm{pc}$

where $D_{\mathrm{d}}, D_{\mathrm{s}}$ and $D_{\mathrm{ds}}$ are angular diameter distances to the deflector, the source and between deflector and source respectively, and $M$ is the mean mass of all microlenses. The duration of crossing the Einstein radius will be up to 10 years assuming a relative velocity between source and lens that is equal or larger than the velocity in the lensing galaxy which is of the order of $\sim(100-400) \mathrm{km} \mathrm{s}^{-1}$ (Mould et al. 1993). Although shorter microlensing events, such as a caustic crossings, can occur, the long time scale 


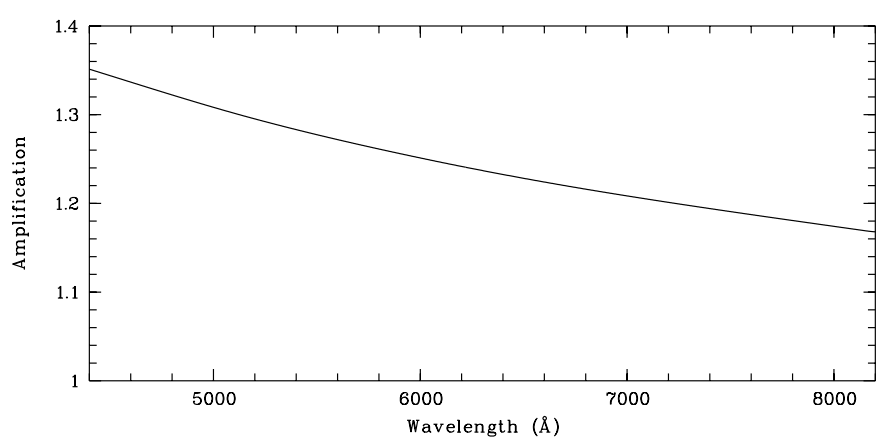

Fig. 11. The residual magnification $\mu$ of $\mathrm{A}$ relative to $\mathrm{B}$ as a function of wavelength. This magnification factor is derived by fitting the $A / B$ continuum ratio after correcting the $\mathrm{B}$ spectrum by the constant factor 3.7 (as in the lower panel of Fig. 7).

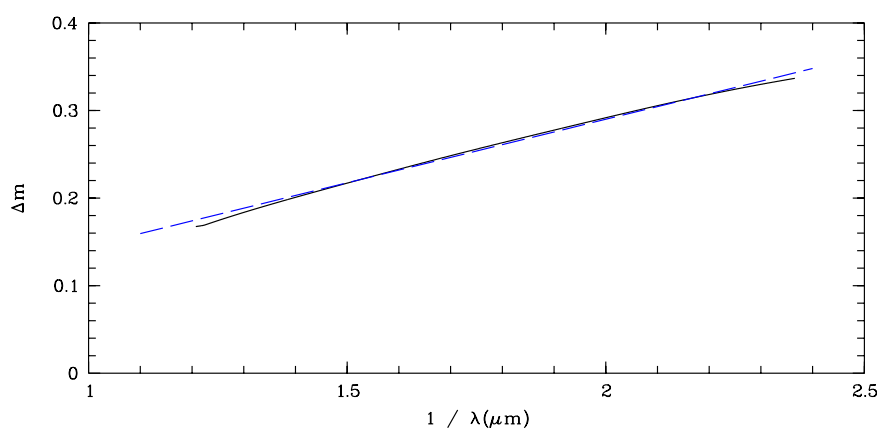

Fig. 12. The magnification of component $A$ in magnitude $(\Delta m=2.5 \log \mu)$ as a function of inverse wavelength (solid line). The wavelength dependence is well reproduced by $\Delta m=$ $0.145 / \lambda(\mu \mathrm{m})$ (dashed line).

of crossing the Einstein radius shows that an event may be present in our data, and stable over our relatively short period of observation of 900 days, as Fig. 9 would suggest.

Assuming that the continuum of $\mathrm{A}$ is amplified by microlensing, we may derive the wavelength dependence of the magnification from the $A / B$ continuum ratio. This relative magnification factor $\mu$ is given in Fig. 11. It is derived by fitting the $A / B$ continuum ratio after correcting the spectrum of $\mathrm{B}$ by the constant factor 3.7. The relative magnification is directly related to the size of the continuum region as a function of wavelength (e.g. Schneider et al. 1992), the precise measurement of which requires the knowledge of the background magnification $\mu_{0}$ due to other distant subimages. While $\mu$ can be estimated from Fig. $11, \mu_{0}$ can only be obtained by measuring the background magnification before and after a caustic crossing event. This can be done with a spectrophotometric monitoring. In Fig. 12, we have plotted the magnification factor - expressed as a magnitude difference - as a function of the inverse wavelength. It shows a remarkably tight wavelength dependence strikingly similar to that found by Nadeau et al. (1999) for Q2237+0305. This suggests that HE 2149-2745 presently suffers a chromatic microlensing event similar (although of much longer duration) to the 1991 high-magnification event in Q2237+0305 and which was interpreted - on the basis of its colour dependence -

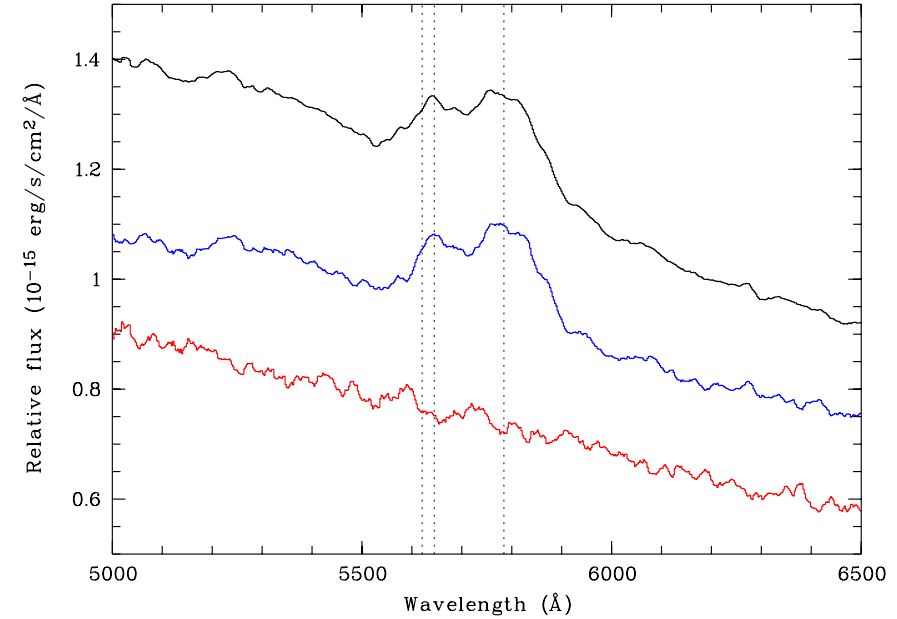

Fig. 13. An enlargement of the spectra of components A (upper) and B (middle, multiplied by 3.7), together with the difference spectrum $A-3.7 \times B$ (lower, multiplied by 2 ). The region of the CIII] emission line is illustrated. The wavelengths of AlIII $\lambda \lambda 1855,1863$ and CIII] $\lambda 1909$ are indicated at the redshift of the quasar $(z=2.03)$. This blend is typical of BAL quasars (Hartig \& Baldwin 1986). Some subtle differences in the blue part of the line profiles may be noticed (see text).

as evidence for the thermal accretion disk model as the source of UV-visible continuum emission in quasars (cf. Nadeau et al. 1999).

While broad emission lines should be essentially unaffected by the caustic crossing, some subtle differences may arise in the line profiles if some parts of the BELR are selectively magnified (Nemiroff 1988; Schneider \& Wambsganss 1990). Looking in detail at the line profiles of components A and B (Fig. 13), small differences are indeed present in the blue wing of the AlIII and CIII] emission lines. Interpreted in terms of microlensing, this could indicate that part of the BELR is being magnified. Similar profile variations are predicted by the models of Schneider \& Wambsganss (1990). Interestingly, no difference in the CIV BAL profile, like those reported for the quadruple BAL quasar H1413+117; cf. Angonin et al. (1990) and Hutsemékers (1993), is noticed.

The present findings may be of importance for investigating the inner structure of quasars. Spectroscopic data obtained at regular time intervals, e.g., spectra separated by intervals corresponding to the time-delay, will test the microlensing interpretation, cancel possible time-delay effects (see Wisotzki et al. 1995) and help to probe the innermost region of quasar structure, using microlensing magnification.

\subsubsection{Reddening}

An alternative explanation for the observed colour difference between quasar images is that the B image is reddened by the lensing galaxy. Assuming this is true, we can estimate the amount of reddening which would correspond to the strength of the putative $\mathrm{Ca} \mathrm{H} \& \mathrm{~K}$ absorption 
lines seen in the B spectrum (Fig. 8), if average Galactic conditions apply to the present case. From the interstellar line observations of Sembach et al. (1993), we derive a mean relation between $E(B-V)$ and the Ca K equivalent width. With a de-redshifted equivalent width $\sim 100 \mathrm{~m} \AA$, this would imply a colour excess $E(B-V) \sim 0.05$, which corresponds to $E(V-I) \sim 0.1$, using the extinction curves of Mathis (1990), after proper redshift corrections are applied. This is compatible with the measured colour difference of 0.15 (Fig. 9). The extinction can also be estimated by fitting the magnitude difference of the $\mathrm{A}$ and $\mathrm{B}$ components as a function of wavelength (Fig. 12) with a typical extinction law. In the wavelength range of interest, the Galactic and SMC extinction curves are very similar (Pei 1992) and can be reasonably well represented by

$A_{\lambda}=\frac{1.75 E(B-V)}{\lambda(\mu \mathrm{m})}$

where $A_{\lambda}$ is the extinction in magnitudes, and where the ratio of the total-to-selective extinction $R_{V}$ is taken to be $R_{V}=3.1$. Identifying the wavelength dependence of the magnitude difference in Fig. 12 to this extinction curve at the redshift of the lens, we have $1.75 E(B-V)\left(1+z_{\text {lens }}\right)=$ 0.145 i.e., $E(B-V)=0.05$ which is in agreement with what is found from the $\mathrm{Ca} \mathrm{H} \& \mathrm{~K}$ lines.

In the case of extinction by dust in the lens we would expect the continuum and the BELR to be equally affected. This does not seem to be the case; however, the wavelength range between CIV and the CIII]-AlIII complex is small. A more sensitive test of the dust hypothesis will be to observe $\mathrm{H}$-alpha in the infra-red, as this will provide a larger wavelength baseline to measure differences.

On the basis of the available data, no interpretation of the apparent reddening can be excluded, although microlensing seems a somewhat more natural explanation for the observed spectral differences.

\section{Lens modeling}

The time-delay measured for HE 2149-2745 can be used to infer an estimate of $H_{0}$, based on modeling of the total gravitational potential responsible for the lensing effect. This includes the main lensing galaxy and any intervening massive cluster along the line of sight to the quasar. HE $2149-2745$ is a relatively easy case to model, as there is no obvious mass concentration along the line of sight to the source apart from the main lensing galaxy. Only a marginally detected galaxy concentration is seen $30^{\prime \prime}$ West and $1^{\prime}$ North of the main lens. In addition, the lensing galaxy is almost aligned with the two quasar images (see Figs. 1 and 14). Two different approaches can be used to convert time delays into $H_{0}$ : models that involve an analytical form for the lensing galaxy, and models that involve more degrees of freedom, e.g., a pixelated surface mass density (Saha \& Williams 1997; Williams \& Saha 2000). We use both approaches.

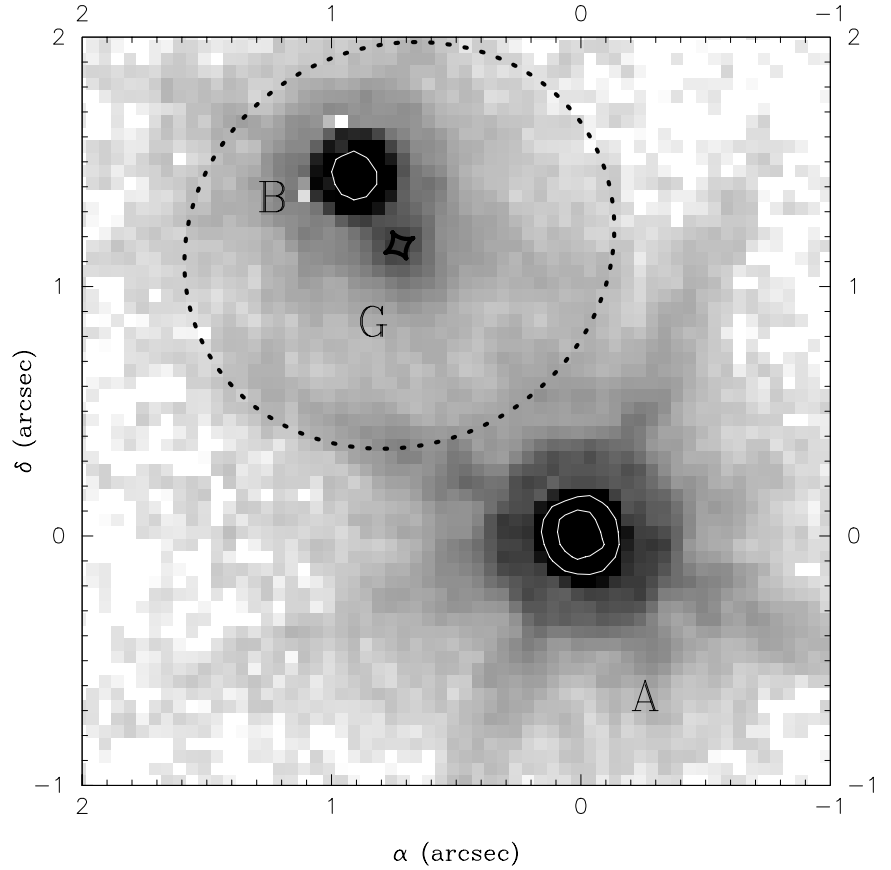

Fig. 14. Field of $3^{\prime \prime}$ around HE 2149-2745, where the lensing galaxy is indicated as $\mathrm{G}$, close to the faint quasar image B. Also indicated are the critical and caustic curves created by a single lensing galaxy and no external shear. The image has been obtained from the CASTLEs public survey of gravitational lenses. This $I$-band image comes from HST/WFPC2 (Kochanek et al.).

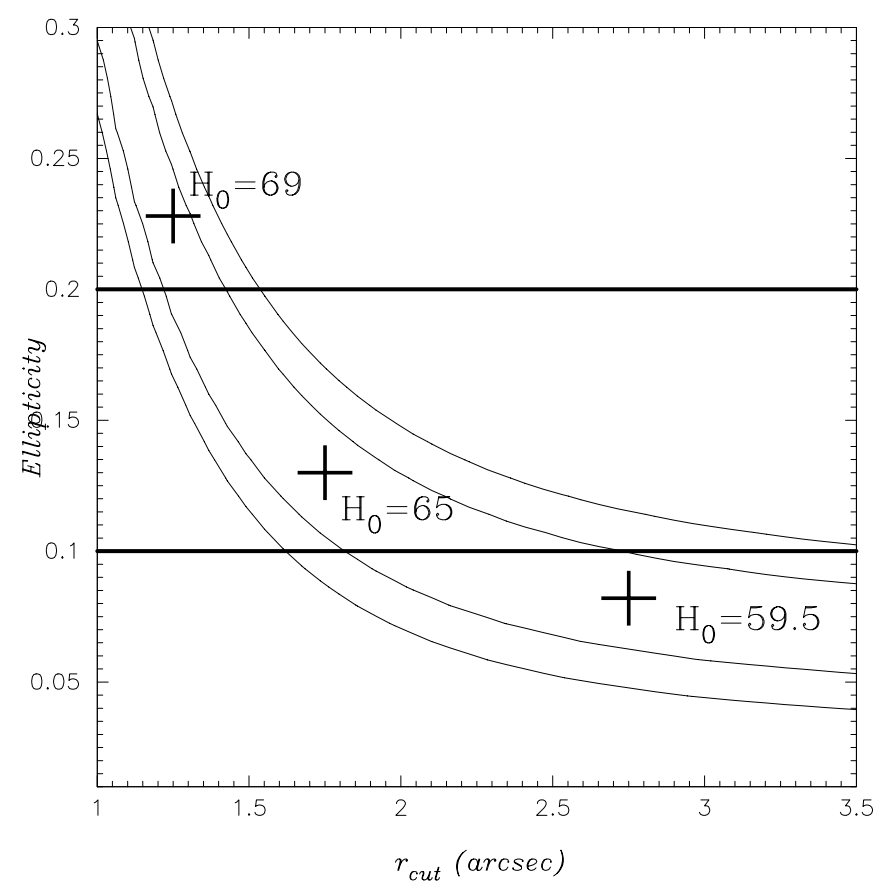

Fig. 15. Results of the model fitting when exploring the ellipticity/cut radius space. The lines show the best models, i.e., they show the area where the models fit the data at $1 \sigma$ (inner lines) and $3 \sigma$ (outer lines). Values for $H_{0}$ are given for different models among the most likely. The horizontal lines give the plausible range of ellipticities, given the HST images. The plot displayed here uses $\Omega=0.3, \lambda=0.7$. 


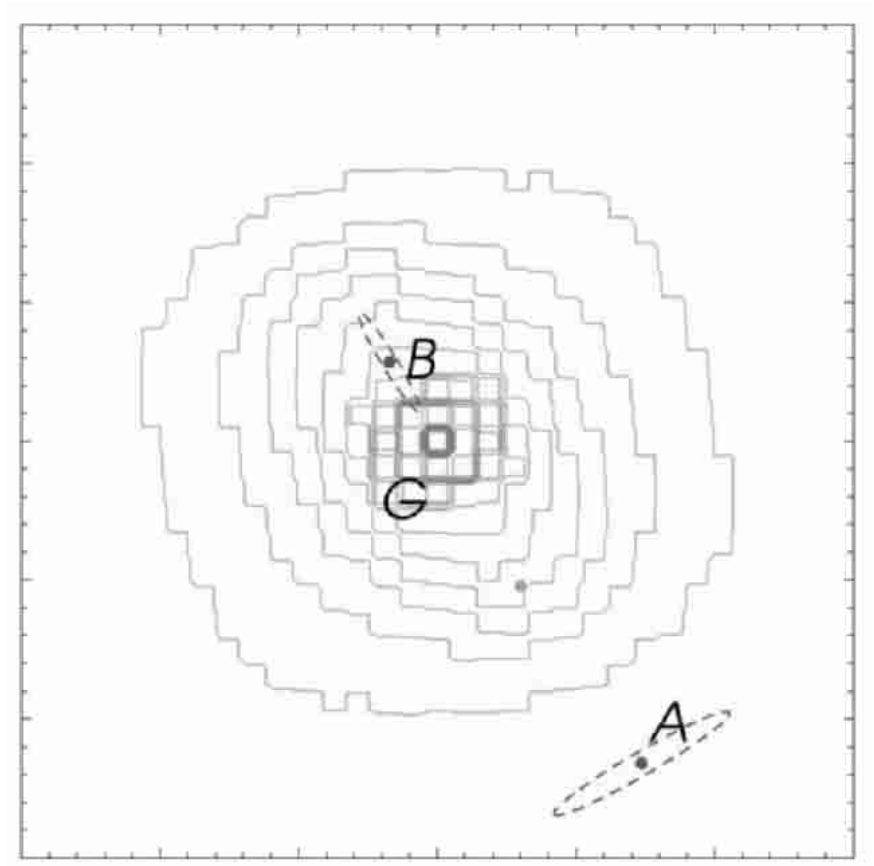

Fig. 16. Mass distribution found with the pixelated models with cosmology $\Omega=0.3, \lambda=0.7$. Almost no ellipticity is found. The contour levels represent $\kappa=1 / 3,2 / 3,1$, etc. The scale is $3^{\prime \prime}$ on a side and the astrometry is the same as for the analytical models. The dashed ellipses around the quasar images represent the magnification matrix, i.e., the axes are proportional to the eigenvalues and the orientation corresponds to the eigenvectors. The spot between the quasar images is the position of the lensed source in the source plan.

\subsection{Analytical models}

Our analytic model uses the mass profile of Kneib et al. (1996): a truncated Pseudo-Isothermal-Elliptical-MassDistribution (PIEMD). Truncated PIEMD are elliptical mass distributions smoothly truncated at radius $r_{\text {cut }}$. For radii smaller (respectively larger) than $r_{\text {cut }}$, the projected surface mass density profile is varying as $r^{-1}$ (respectively $r^{-3}$ ). The interest in such profile is their ability of characterizing any ellipticity in the mass distribution as well as having a total finite mass. We fit the model to the publicly available HST/WFPC2 data (which offers better resolution and sampling than NICMOS) obtained by Kochanek et al., and use the emission line flux ratio of 3.7 calculated from our VLT spectra. During the fit, the astrometry of the quasar images relative to the lens is fixed, as well as the redshift of the source and lens. The free parameters include the velocity dispersion, ellipticity, which is here defined as $\left[a^{2}-b^{2}\right] /\left[a^{2}+b^{2}\right]$ where $a$ and $b$ are the long and short axes of the lens, and cut radius of the lens.

A common problem in lens modeling is the mass-sheet degeneracy. In other words, several mass profiles and in particular mass profiles of different compactness, will reproduce the same time-delay (Gorenstein et al. 1988). One has therefore to constrain the models using observational data and explore the widest range of physically acceptable models. A first constraint comes from the HST
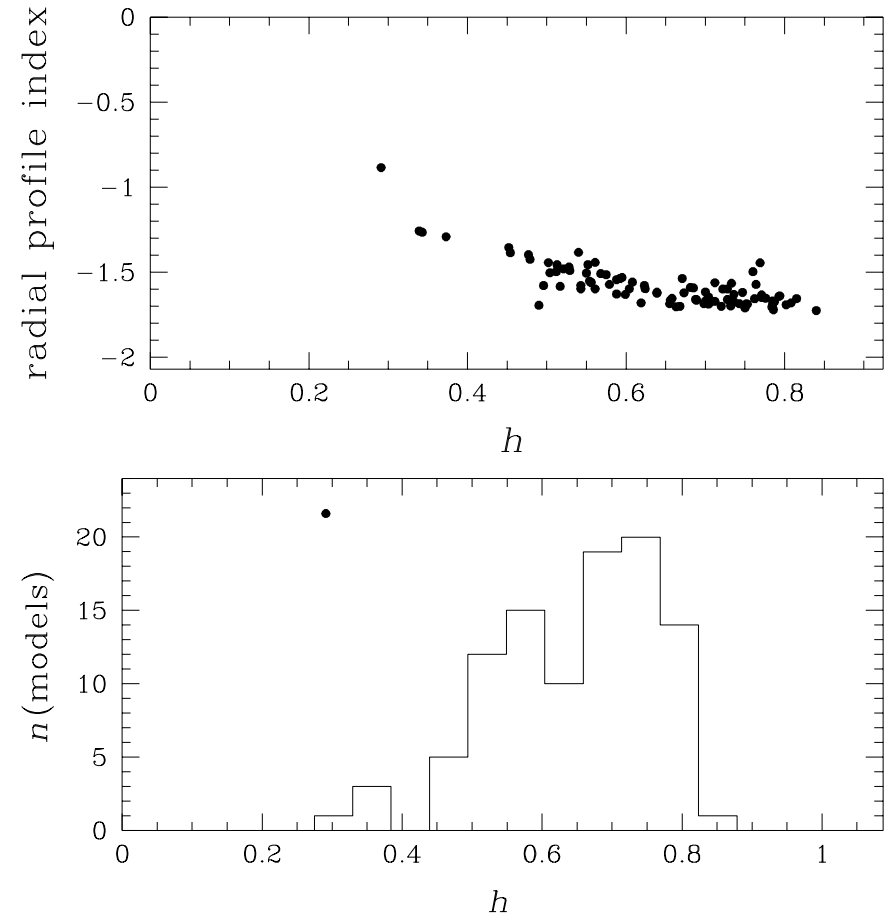

Fig. 17. Top: radial index profile vs. $H_{0}$ for one hundred different non-parametric models, showing that all the galaxy models have a steep mass profile $(<-0.5)$. Bottom: probability density for $H_{0}$ for the same models. Units on the $X$ axis of both figures is in $h_{100}$. The median value of the distribution is $65 \mathrm{~km} \mathrm{~s}^{-1} \mathrm{Mpc}^{-1}$. The black dot marks the location of an isothermal mass profile (radial profile index $=-1$ ).

images: the lens is almost round. After PSF-subtraction of the quasar images we fit the shape of the lensing galaxy and obtain an ellipticity of about 0.1-0.2. Assuming that the mass distribution follows the light distribution we can restrict ourselves to this range of ellipticities. Figure 15 shows a family of models exploring the ellipticity vs. $r_{\text {cut }}$ space and we see that little spread in the values of $H_{0}$ is observed in the range of ellipticities $0.1-0.2$. We assume ellipticity $\varepsilon=0.15$ which implies $r_{\text {cut }}=1.6^{\prime \prime}$ and $H_{0}=66 \pm 8 \mathrm{~km} \mathrm{~s}^{-1} \mathrm{Mpc}^{-1}$ with additional systematic errors of $\pm 3 \mathrm{~km} \mathrm{~s}^{-1} \mathrm{Mpc}^{-1}$ due to the limited range of ellipticity. We also try to investigate the existence of a galaxy core. For this we scan the possible values for the core radius. The lens model excludes any value of $r_{\text {core }}$ larger than 0.02 arcsec. Within this limit, the core radius has basically no effect on the determination of $H_{0}$, so the choice of a given core radius is not critical. In our fiducial model, we use a core radius of $\sim 0.002$ arcsec which corresponds to $\sim 10 \mathrm{pc}$. Using this, and keeping the other parameters free, we find a lens with the parameters summarized in Table 2. We show in Table 3 the effect of a change in cosmology. Due to the relatively low redshift of the lensing galaxy the influence of a change in cosmology is small compared to the measurement errors.

If we take the currently most popular cosmology, i.e., $\Omega=0.3, \lambda=0.7$, and $\Delta t=103 \pm 12$ days, we find $H_{0}=66 \pm 8 \mathrm{~km} \mathrm{~s}^{-1} \mathrm{Mpc}^{-1}$ with an additional estimated 
Table 2. Model parameters for HE 2149-2745. The values between brackets are fixed during the fit, the others are fitted. The core radius is negligible.

\begin{tabular}{lc}
\hline Parameter & Fit \\
\hline$\Omega, \lambda$ & {$[0.3,0.7]$} \\
Ellipticity & $0.13 \pm 0.05$ \\
PA (degrees) & $62 \pm 5$ \\
Vel. disp. $\left(\mathrm{km} \mathrm{s}^{-1}\right)$ & $198 \pm 10$ \\
$r_{\text {cut }}$ & {$\left[1.25^{\prime \prime}\right]$} \\
Core radius & {$\left[0.002^{\prime \prime}=0.01 \mathrm{kpc}\right]$} \\
time-delay & {$[103$ days $]$} \\
\hline
\end{tabular}

Table 3. Influence of the cosmology on the modeling of HE 2149-2745. Values of $H_{0}$ are listed for an Einstein-deSitter Universe $(\Omega=1.0, \lambda=0$. $)$, an open Universe $(\Omega=0.3$, $\lambda=0$.) and a flat Universe with non-zero cosmological constant $(\Omega=0.3, \lambda=0.7)$. The model parameters are fixed and set to the ones in Table 2 .

\begin{tabular}{cr}
\hline Cosmology & $H_{0}\left(\mathrm{~km} \mathrm{~s}^{-1} \mathrm{Mpc}^{-1}\right)$ \\
\hline$\Omega=0.3, \lambda=0.7$ & $66 \pm 8$ \\
$\Omega=0.3, \lambda=0.0$ & $67 \pm 8$ \\
$\Omega=1.0, \lambda=0.0$ & $61.5 \pm 8$ \\
\hline
\end{tabular}

systematic error of $\pm 3 \mathrm{~km} \mathrm{~s}^{-1} \mathrm{Mpc}^{-1}$ due to parameter choices.

\subsection{Pixelated models}

The pixelated models of Saha \& Williams (1997) and Williams \& Saha (2000) were also applied to HE 2149-2745. While the mass distribution resulting from these models is not as well related to the physical parameters of the lens, they allow us to explore a wide range of lens shapes and to estimate the robustness of the time-delay conversion. Figure 16 shows the mass distribution found by the models. During the fit, the ellipticity of the mass distribution was kept free. The program reconstructs almost round mass distributions, as does the analytical model (Fig. 15). We also examine the impact of a change in the compactness of the mass profile on the derived value for $H_{0}$. Figure 17 shows the result of a Monte-Carlo simulation where 100 models were run with different indices for the lens shape. The density index in Fig. 17 represents the logarithmic projected density gradient in the vicinity of the images (cf. Williams \& Saha 2000). The upper panel of the figure shows that most models predict relatively steep/concentrated mass profiles.

In the lower panel of Fig. 17 we display the $H_{0}$ probability distribution. The mean of the distribution peaks at $H_{0}=65 \pm 15 \mathrm{~km} \mathrm{~s}^{-1} \mathrm{Mpc}^{-1}$ at the $2-\sigma$ level and $H_{0}=65 \pm 8 \mathrm{~km} \mathrm{~s}^{-1} \mathrm{Mpc}^{-1}$ at the 1- $\sigma$ level.

\section{Summary - conclusions}

We have presented the first result of a long-term photometric monitoring campaign undertaken at ESO between 1998 and 2000 with the 1.54-m Danish telescope. Our $V$ and $i$ light curves allow us to measure a time-delay of $\Delta t=103 \pm 12$ days between the two quasar images of HE 2149-2745.

From VLT spectroscopy, we have derived a tentative estimate of the lens redshift to be $z=0.489$. Applying both analytic and numerical lens models to the case of HE 2149-2745, we derive $H_{0}=66 \pm 6 \mathrm{~km} \mathrm{~s}^{-1} \mathrm{Mpc}^{-1}$ with an additional systematic error of $\pm 3 \mathrm{~km} \mathrm{~s}^{-1} \mathrm{Mpc}^{-1}$ in the case of the analytic models, due the limited range of the ellipticity. The derived mass models are relatively compact, although not as compact as the light profile of the galaxy. An extra source of systematics might be introduced by the uncertainty on the lens redshift estimate. This is however not critical for the determination of $H_{0}$ as the error is dominated by the uncertainty in the time-delay measurement. As HE 2149-2745 shows smooth light curves, it is likely that the situation can be improved by continued monitoring. With an improved time-delay it would also be highly desirable to re-determine the lens redshift more precisely.

Our monitoring program of HE 2149-2745 is the first to be carried out in two bands on such a regular basis and over such a long time scale. Given the error bars, we do not see any significant colour variation over the 900 days of observation. Moreover, our spectra of the two quasar images show that the flux ratios in the broad emission lines behave differently for the continuum flux ratio, and that the flux ratio measured in the BAL structure of the source follows the behavior as the continuum region. Such behavior can be explained both by microlensing or by differential extinction by the lensing galaxy, or both. So far, the data do not allow us to distinguish between the two possible explanations. In order to confirm microlensing, one would need for example to know the time-scale of the putative event, i.e., to measure the absolute magnification of the event and its duration. This would not only allow us to confirm microlensing, but also to use it to map the radial structure of the central AGN in the source. Although the Einstein radius crossing time is long for HE 2149-2745, of the order of 10 years, much shorter microlensing events, such as a caustic crossings, can occur. Conducting a longterm spectrophotometric monitoring could therefore allow us to probe the AGN size HE 2149-2745.

Acknowledgements. We thank IJAF and ESO for granting us observing time for this project on a flexible basis. We are very grateful to the $2.2-\mathrm{m}$ team for their support and enthusiasm. Most of the monitoring observations presented here have been carried out during short periods of time allocated during the scheduled runs of regular observers. We appreciated very much the enthusiasm of all the observers who accepted to perform the observations for us. Some of them, present at the telescope for long periods, contributed a lot to our program. It is a pleasure to thank in particular B. Milvang-Jensen, L. Fogh-Olsen, S. Frandsen, L. Hansen, H. Kjeldsen, J. Knude and G. Israel. We thank Lutz Wisotzki for letting us use his EFOSC spectrum and Prasenjit Saha for help with the non-parametric models. IB was supported by Pôle d'Attraction Interuniversitaire, P4/05 (SSTC, 
Belgium). FC acknowledges financial support through Chilean grant FONDECYT/3990024. Additional support from the European Southern Observatory, through ECOS/CONICYT grant C00U05, CNRS/CONICYT grant 8730 and Marie Curie grant MCFI-2001-00242 are also gratefully acknowledged. JH is supported by the Danish Natural Science Research Council (SNF). JPK thanks CNRS for support.

\section{References}

Angonin, M. C., Remy, M., Surdej, J., \& Vanderriest, C. 1990, A\&A, 233, L5

Burud, I., Hjorth, J., Jaunsen, A. O., et al. 2000, ApJ, 544, 117

Courbin, F., Magain, P., Kirkove, M., \& Sohy, S. 2000a, ApJ, 539,1136

Courbin, F., Lidman, C., Meylan, G., Kneib, J.-P., \& Magain, P. 2000b, A\&A, 360, 853

Fassnacht, C. D., Pearson, T. J., Readhead, A. C. S., et al. 1999, ApJ, 527, 498

Gorenstein, M. V., Shapiro, I. I., \& Falco, E. E. 1988, ApJ, 327,693

Hartig, G. F., \& Baldwin, J. A. 1986, ApJ, 302, 64

Hjorth, J., Burud, I., Jaunsen, A. O., et al. 2001, in Gravitational Lensing: Recent progress and future goals, ed. T. Brainerd, \& C. Kochanek, ASP Conf. Ser., in press

Hutsemékers, D. 1993, A\&A, 280, 435

Kinney, A. L., Calzetti, D., Bohlin, R. C., et al. 1996, ApJ, 467, 38

Kneib, J.-P., Ellis, R. S., Smail, I., Couch, W. J., \& Sharples, R. M. 1996, ApJ, 471, 643

Kochanek, C. S., Falco, E. E., Impey, C., et al. http://cfa-www.harvard.edu/glensdata/

Kochanek, C. S., Falco, E. E., Impey, C. D., et al. 2000, ApJ, 543,131

Koopmans, L. V. E., de Bruyn, A. G., Xanthopoulos, E., \& Fassnacht, C. D. 2000, A\&A, 356, 391

Krolik, J. 1999, in Active galactic nuclei: From the central black hole to the galactic environment. Princeton Series in Astrophysics, ed. J. P. Ostriker (Princeton University Press)

Kurtz, M. J., \& Mink, D. J. 1998, PASP, 110, 934

Lidman, C., Courbin, F., Kneib, J.-P., et al. 2000, A\&A, 364, L62

Magain, P., Courbin, F., \& Sohy, S. 1998, ApJ, 494, 452

Mathis, J. S. 1990, ARA\&A, 28, 37

Mould, J. R., Akeson, R. L., Bothun, G. D., et al. 1993, ApJ, 409, 14

Nadeau, D., Racine, R., Doyon, R., \& Arboit, G. 1999, ApJ, 527,46

Nemiroff, R. J. 1988, ApJ, 335, 593

Osterbrock, D. E. 1989, Astrophysics of Gaseous Nebulae and Active Galactic Nuclei (Universtiy Science Books)

Pei, Y. C. 1992, ApJ, 395, 130

Refsdal, S. 1964, MNRAS, 128, 307

Saha, P., \& Williams, L. L. R. 1997, MNRAS, 292, 148

Schechter, P. L., Bailyn, C. D., Barr, R., et al. 1997, ApJ, 475, L85

Schild, R. E. 1990, AJ, 100, 1771

Schneider, P., \& Wambsganss, J. 1990, A\&A, 237, 42

Schneider, P., Ehlers, J., \& Falco, E. E. 1992, Gravitational lenses (Springer, Berlin)

Sembach, K. R., Danks, A. C., \& Savage, B. D. 1993, A\&AS, 100,107

Tonry, J., \& Davis, M. 1979, AJ, 84, 1511

Vanderriest, C., Schneider, J., Herpe, G., et al. 1989, A\&A, 215,1

Wambsganss, J., Schneider, P., \& Paczynski, B. 1990, ApJ, 358, L33

Wambsganss, J., \& Paczynski, B. 1991, AJ, 102, 854

Williams, L. L. R., \& Saha, P. 2000, AJ, 119, 439

Wisotzki, L., Koehler, T., Kayser, R., \& Reimers, D. 1993, A\&A, 278, L15

Wisotzki, L., Koehler, T., Ikonomou, M., \& Reimers, D. 1995, A\&A, 297, L59

Wisotzki, L., Koehler, T., Lopez, S., \& Reimers, D. 1996, A\&A, 315, L405 